\title{
The influence of compression therapy on the levels of inflammatory biomarkers in patients with chronic venous disease
}

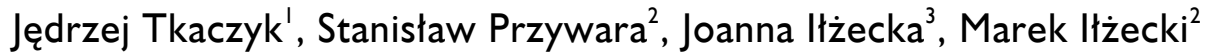 \\ 'Doctoral School, Department of Vascular Surgery and Angiology, Medical University of Lublin, Poland \\ ${ }^{2}$ Department of Vascular Surgery and Angiology, Medical University of Lublin, Poland \\ ${ }^{3}$ Independent Neurological Rehabilitation Unit, Medical University of Lublin, Poland
}

\begin{abstract}
Chronic venous disease (CVD) is defined as any morphological and functional abnormalities of long duration manifested either by symptoms and/or signs indicating the need for investigation and/or care. The pathophysiological mechanism of CVD can be characterized by reflux, obstruction, or a combination of both, which leads to increased venous pressure.

Compression therapy remains the gold standard of the conservative treatment of CVD in all stages. The possible forms of compression therapy are elastic stocking, non-elastic and elastic bandages, and intermittent pneumatic compression. Compression bandages have been proven to improve the healing of venous ulcers, in comparison with standard care without compression therapy.

In the last years, inflammation has been shown to play an important role in the pathophysiology of CVD. The influence of the altered shear stress on the endothelial cells (EC) causes EC to release inflammatory molecules, chemokines, vasoactive agents, express selectins, and prothrombotic precursors such as ICAM-I, MCP-I I MIP I $\beta$, VCAM, L-selectin, E-selectin, IL-I $\beta$, IL-4, IL-6, IL-8, IL-I2p40, IL-13, G-CSF, GM-CSF, IFN- $\gamma$, TNF- $\alpha$, and $M I P-\mid \alpha$

Several studies have been performed to investigate the influence of compression therapy on the level of various inflammatory biomarkers in patients with CVD. In these studies level of the most inflammatory molecules, such as IL-I $\beta, I L-6, I L-8, I L-12 p 40$, G-CSF, GM-CSF, IFN- $\gamma$, TNF- $\alpha$, VEGF, MMP 3, 8, 9 and TIMP-I decreased after the therapy.
\end{abstract}

Key words: inflammatory biomarkers, compression therapy, chronic venous disease, inflammation

Acta Angiol 2021; 27, I: 32-36

\section{Introduction}

Chronic venous disease (CVD) is defined as any morphological and functional abnormalities of long duration manifested either by symptoms and/or signs indicating the need for investigation and/or care [I]. The prevalence of CVD has been previously investigated in many epidemiological studies that have been carried all around the world. The reported incidence of CVD ranges between $20 \%$ and $60 \%$, depending on the region where the research was conducted, different criteria for patient selection, disease definition, different imaging techniques used, and exposure to risk factors [2]. The reported prevalence of CVD was higher in the western populations of developed regions that are exposed to various risk factors such as sedentary lifestyle, low fiber diet, or constipation [3]. More advanced stages of CVD (C3-C6 in the CEAP classification) affect about 5\% of the population, and the end stages $\left(\mathrm{C}^{-} \mathrm{C} 6\right)$ of the CVD - about I-2\% [4]. The presence of CVD may be associated with lower quality of life - almost $30 \%$ of patients present with symptoms of depression [5]. 


\section{Pathophysiology of CVD}

The pathophysiological mechanism of CVD can be characterized by reflux, obstruction, or a combination of both. Both lead to increased ambulatory venous pressure. Other factors may be e.g. failure of the calf and foot muscle pomp (in neuromuscular problems) [5]. The most frequent cause of venous hypertension is valvular incompetence $(70-80 \%$ of cases), while isolated outflow obstruction is found in ca. $2 \%$. Valvular incompetence may be secondary to deep vein thrombosis in $18-25 \%$, or due to congenital anomaly in $\mathrm{I}-3 \%[6]$

\section{The role of inflammation in a CVD}

In the last years, inflammation has been shown to play an important role in the pathophysiology of CVD [7]. Venous hypertension in the lower limbs leads to a vicious circle of vascular and inflammatory phenomena that increases hypertension even more. High venous pressure in the lower extremities leads to leukocyte accumulation due to leukocyte adhesion to the endothelium and migration through the endothelium of small vessels [8]. It also results in a reduction of shear stress (SS) the main regulator of endothelial activation state that promotes pathological changes of venous valves and vein wall [9]. Low SS leads to local inflammation, through the activation of endothelial cells (ECs) and leukocytes, that release vasoactive agents, express selectins, inflammatory molecules, chemokines, and prothrombotic precursors, and through the infiltration of inflammatory cells into venous walls and leaflets $[10,1 \mathrm{I}]$. Changes in EC signaling result in increased production of inflammatory biomarkers, e.g. chemokines, cytokines, growth factors, proteases, and others, that cause worsening of the inflammatory process [12]. Many studies report an increased expression of inflammatory markers in vitro, in pre-clinical studies, and in patients with CVD, which confirms the role of inflammation in CVD [13].

\section{Compression therapy - a gold standard in a treatment of CVD}

Compression therapy remains the gold standard of the conservative treatment of CVD in all stages, because of its non-invasive nature, ease of use, and efficacy of reducing venous hypertension which is a main pathophysiological mechanism of CVD. The possible forms of compression therapy are elastic stocking, non-elastic and elastic bandages, and intermittent pneumatic compression [5].
In the last decades, elastic stockings have been the cornerstone of conservative treatment of C0-C4 CVD. Despite their popularity, evidence of the efficacy for this type of compression remains unclear and is based on the lack of randomized controlled trials for both primary and post-thrombotic CVD [14, 15]. However, there is some evidence, based on non-RCTs and clinical experience supporting their use to improve patients' symptoms [15] and quality of life [16].

Compression bandages have been proven to improve the healing of venous ulcers, in comparison with standard care without compression therapy [17]. Types of bandages include traditional systems with elastic components and non-elastic compression systems [18]. High compression systems with a sustained compression of at least $40 \mathrm{~mm} \mathrm{Hg}$ with a four-layer bandage have been shown to be more effective than the lower grades of compression [19]. Compression bandages are recommended as the initial treatment in patients with venous leg ulcers [5].

\section{Inflammatory biomarkers in patients with CVD}

In the last years, many studies confirmed an increased level of proinflammatory molecules in patients with CVD. The influence of the altered shear stress on the endothelial cells (EC) causes EC to release inflammatory molecules, chemokines, vasoactive agents, express selectins, and prothrombotic precursors [20]. ICAM-I is a molecule responsible for the detection of changes in mechanical stress forces and SS, promoting leukocyte recruitment, adhesion, and transmigration [2I]. Overexpression of ICAM-I in EC has been shown in several studies $[22,23]$. MCP-I, macrophage inflammatory protein $\mathrm{I} \beta$, vascular cell adhesion molecule (VCAM), as well as L-selectin, E-selectin, and ICAM-I enhance leukocyte rolling, adhesion, and migration through the endothelium of vein wall and valve [2I]. Plasma levels of VCAM-I, angiotensin-converting enzyme, endothelial leukocyte adhesion molecule, and L-Selectin are also increased in varicose veins $[24,25]$.

The endothelial glycocalyx (GCX) - structure responsible for the prevention of leukocyte adhesion, thrombosis, and inflammation in CVD is injured by the altered shear stress and mechanical forces on the wall of the vein, which leads to loss of GCX [26]. In the vein wall of varicose veins, there is an increased level of degraded, sulfated glycosaminoglycans, which confirms the GCX disruption [27].

Matrix metalloproteinases - proteolytic enzymes identified in many tissues and organs including the venous system, play a crucial role in tissue remodeling 
and turnover of the collagen, elastin, and other proteins [28]. Overexpression of MMPs and cytokines has an important effect on the venous wall and valves causing tissue destruction with skin changes and formation of the ulcer [29,30]. In vein samples from the patients with CVD, have been found a variety of MMPs such as: MMP-I, $-2,-3,-8,-9,-12$, and -13 [3I]. Their role includes degradation of adventitial extracellular matrix, as well as collagen bundles and elastin in the medial layer, having also potential early effect on venous dilation [32]

Cytokines were proven to play an important role at different stages of CVD. The majority of pro-inflammatory cytokines (IL-I $\beta$, IL-4, IL-6, IL-8, IL- I 2p40, IL- I 3, G-CSF, GM-CSF, MCP-I, IFN- $\gamma$, TNF- $\alpha$, MIP-I $\alpha$, and MIP-I $\beta$ ) were found to be elevated in ulcer tissue from patients with CVD [33].

\section{Levels of inflammatory biomarkers in patients undergoing compression therapy}

Several studies were performed to investigate the influence of compression therapy on the levels of various inflammatory biomarkers in patients with CVD.

The interesting study held by Beidler et al. investigated changes in inflammatory cytokine levels in ulcer tissue from patients with CVD treated with a high-grade 3- or 4-layer compression bandage system for 4 weeks. The study group consisted of 30 limbs with untreated CVD and leg ulceration. Tissue samples were obtained using biopsy from healthy and ulcerated tissue before and after therapy. Cytokine levels were measured using a multiplexed protein assay. The majority of pro-inflammatory cytokine levels (IL-I $\beta$, IL-6, IL-8, IL- I 2p40, IL- I 3, G-CSF, GM-CSF, MCP-I, IFN- $\gamma$, TNF- $\alpha$, MIP-I $\alpha$, MIP-I $\beta$ ) were elevated in ulcer tissue, in comparison with controls. Most of the investigated molecule levels (IL-I $\beta$, IL-6, IL-8, IL-I 2p40, G-CSF, GM-CSF, IFN- $\gamma$, TNF- $\alpha$ ) significantly decreased after the compression therapy. On the other hand, the level of an anti-inflammatory cytokine, IL- I Ra, increased after 4 weeks of treatment. Ulcers with a higher level of IL-I $\alpha$, IL-I $\beta$, IFN- $\gamma$, IL-I 2 p40, and GM-CSF were more likely to heal rapidly [33].

In an earlier study, Murphy et al. investigated serum levels of VEGF and TNF- $\alpha$ in a group of 8 patients undergoing 4 weeks of treatment with 4-layered, graduated compression therapy. Serum samples were taken from the superficial veins of the lower limb before and after the treatment. Serum from the arms of the same patients was used as a control. The level of VEGF and TNF- $\alpha$ were measured with ELISA assay. All patients had elevated cytokine levels before the treatment compared with the control. In each case, the reduction to below control values in the levels of VEGF and TNF- $\alpha$ was observed [34].
Another study, held by Gohel et al. [35], comprised 80 patients with chronic leg ulceration treated with multilayer compression bandaging. Inflammatory biomarkers were measured in the wound fluid and in the venous blood taken from the antecubital vein at recruitment and after 5 weeks as well as the size of ulceration. Levels of proinflammatory molecules were measured using the ELISA technique. Median ulcer size reduced from $4.4 \mathrm{~cm}^{2}$ to $2.2 \mathrm{~cm}^{2}$ after the treatment. The volume of wound fluid strongly correlated with the size of an ulcer. Among all of the investigated cytokines and factors (TNF $\alpha$, ILI $\beta$, bFGF, VEGF, TGF $\beta$ I , MMP2, MMP9) only bFGF showed a significant positive correlation between its concentration in wound fluid and an ulcer size. Changes in wound fluid $\operatorname{TGF} \beta$ I concentrations inversely correlated with changes in ulcer size. Other factors did not show significant correlations with ulcer healing. Also, the correlation between the wound fluid and serum cytokine concentrations was poor [35].

In a 2008 study, Beidler et al. [36] investigated levels of matrix metalloproteinases (MMPs) in a leg ulcer tissue of patients with CVD before and after 4 weeks of high-strength compression therapy. The study group included 29 patients with untreated CVD and leg ulceration. Tissue samples were obtained by biopsies from healthy tissues and from ulcerated tissue before and after therapy. MMPs were measured using a multiplexed protein assay. MMPI , 2, 3, 8, 9, I2, and I 3 levels were elevated in ulcer tissue comparing with healthy tissue. Levels of MMP3, 8, and 9 significantly decreased after the compression therapy. A decrease in MMPI, 2 and 3 levels was linked with significantly higher rates of ulcer healing at the end of treatment [36].

A more recent study, performed by Caimi et al. investigated plasma concentration levels of gelatinases (MMP-2 and MMP-9) and their inhibitors (TIMP-I and TIMP-2) in a group of 36 patients with CVD and venous leg ulcers, before and after the treatment with a multi-layer bandaging system. The levels of gelatinases and their inhibitors were measured in fasting venous blood using an ELISA kit. A significantly higher level of gelatinases and their inhibitors was observed in patients with leg ulcers, comparing with normal controls. Healing of the ulcers after the therapy was associated with a decrease in MMP-9 and TIMP-I levels and in MMP-2/ /TIMP-2 ratio compared to the baseline values. However, even after the therapy, the levels of all examined parameters were higher than in the control group [37].

\section{Conclusions}

In the last years, inflammation has been proven to play an important role in the pathophysiology of chronic venous disease. 
Compression therapy remains the gold standard of the conservative treatment of CVD in all stages, and has been proven to improve the healing of venous ulcers.

Many studies reported an increased expression of inflammatory markers in vitro, in pre-clinical studies, and in patients with CVD.

Several inflammatory molecules, chemokines, vasoactive agents, selectins, and prothrombotic precursors, such as ICAM-I, MCP-I, macrophage inflammatory protein I $\beta$, VCAM, L-selectin, E-selectin, IL-I $\beta$, IL-4, IL-6, IL-8, IL-I 2p40, IL-I3, G-CSF, GM-CSF, IFN- $\gamma$, TNF- $\alpha$, MIP-I $\alpha$, as well as matrix metalloproteinases MMP-I, $-2,-3,-8,-9,-12$, and -13 were identified in patients with CVD.

Several studies were performed to investigate the influence of compression therapy on the level of various inflammatory biomarkers in patients with CVD.

Presented studies showed a reduction in levels of inflammatory biomarkers such as IL I $\beta$, IL-6, IL-8, IL12p40, G-CSF, GM-CSF, IFN- $\gamma$, TNF- $\alpha$, VEGF, MMP 3 , 8,9 and TIMP-I after the therapy.

Not all biomolecular mechanisms of the healing effect of compression therapy on venous leg ulcers have been well understood, so further studies regarding this topic may be needed.

\section{Conflict of the interest}

None

\section{References:}

I. Eklof Bo, Perrin M, Delis KT, et al. American Venous Forum, European Venous Forum, International Union of Phlebology, American College of Phlebology, International Union of Angiology. Updated terminology of chronic venous disorders: the VEINTERM transatlantic interdisciplinary consensus document. J Vasc Surg. 2009; 49(2): 498-50I, doi: 10.1016/j.jvs.2008.09.014, indexed in Pubmed: 19216970.

2. Zolotukhin IA, Seliverstov EI, Shevtsov YN, et al. Prevalence and Risk Factors for Chronic Venous Disease in the General Russian Population. Eur J Vasc Endovasc Surg. 2017; 54(6): 752-758, doi: 10.1016/j.ejvs.2017.08.033, indexed in Pubmed: 29031868.

3. Robertson L, Evans C, Fowkes FGR. Epidemiology of chronic venous disease. Phlebology. 2008; 23(3): 103-III, doi: 10.1258/ phleb.2007.00706I, indexed in Pubmed: 18467617.

4. Graham ID, Harrison MB, Nelson EA, et al. Prevalence of lower-limb ulceration: a systematic review of prevalence studies. Adv Skin Wound Care. 2003; 16(6): 305-316, doi: 10.1097/00129334-200311000-00013, indexed in Pubmed: 14652517.

5. Wittens C, Davies AH, Bækgaard N, et al. Editor's Choice - Management of Chronic Venous Disease: Clinical Practice Guidelines of the European Society for Vascular Surgery (ESVS). Eur J Vasc Endovasc Surg. 2015; 49(6): 678-737, doi: 10.1016/j. ejvs.2015.02.007, indexed in Pubmed: 2592063।.
6. Labropoulos N. Hemodynamic changes according to the CEAP classification. Phlebolymphology. 2003; 40: 130-136.

7. Grudzińska E, Lekstan A, Szliszka E, et al. Cytokines Produced by Lymphocytes in the Incompetent Great Saphenous Vein. Mediators Inflamm. 2018; 2018: 7161346, doi: 10.1155/2018/7161346, indexed in Pubmed: 30013452.

8. Bergan J. Molecular mechanisms in chronic venous insuffciency. Ann Vasc Surg. 2007; 2I(3): 260-266, doi: 10.1016/j. avsg.2007.03.0II, indexed in Pubmed: 17484957.

9. Anwar MA, Shalhoub J, Lim CS, et al. The effect of pressure-induced mechanical stretch on vascular wall differential gene expression. J Vasc Res. 2012; 49(6): 463-478, doi: 10.1 159/00033915I, indexed in Pubmed: 22796658.

10. Takase S, Bergan JJ, Schmid-Schönbein G. Expression of adhesion molecules and cytokines on saphenous veins in chronic venous insufficiency. Ann Vasc Surg. 2000; 14(5): 427-435, doi: 10.1007/s I00169910092, indexed in Pubmed: 10990550.

II. Castro-Ferreira R, Cardoso R, Leite-Moreira A, et al. The Role of Endothelial Dysfunction and Inflammation in Chronic Venous Disease. Ann Vasc Surg. 2018; 46: 380-393, doi: 10.1016/j. avsg.2017.06.131, indexed in Pubmed: 28688874.

12. Raffetto J. Pathophysiology of Chronic Venous Disease and Venous Ulcers. Surgical Clinics of North America. 2018; 98(2): 337-347, doi: 10.1016/j.suc.2017.11.002.

13. Mansilha A, Sousa J. Pathophysiological Mechanisms of Chronic Venous Disease and Implications for Venoactive Drug Therapy. Int J Mol Sci. 2018; 19(6), doi: 10.3390/ijms 19061669, indexed in Pubmed: 29874834.

14. Azirar S, Appelen D, Prins MH, et al. Compression therapy for treating post-thrombotic syndrome. Cochrane Database Syst Rev. 2019; 9: CD004177, doi: 10.1002/I465I858.CD004I77. pub2, indexed in Pubmed: 31531971.

15. Shingler S, Robertson L, Boghossian S, et al. Compression stockings for the initial treatment of varicose veins in patients without venous ulceration. Cochrane Database Syst Rev. 2013(I2): CD008819, doi: 10.1002/14651858.CD008819.pub3, indexed in Pubmed: 243234II.

16. Andreozzi GM, Cordova R, Scomparin MA, et al. Quality of Life Working Group on Vascular Medicine of SIAPAV. Effects of elastic stocking on quality of life of patients with chronic venous insufficiency. An Italian pilot study on Triveneto Region. Int Angiol. 2005; 24(4): 325-329.

17. Wong IKY, Andriessen A, Charles HE, et al. Randomized controlled trial comparing treatment outcome of two compression bandaging systems and standard care without compression in patients with venous leg ulcers. J Eur Acad Dermatol Venereol. 2012; 26(I): 102-1 10, doi: 10.1 I II/j. 1468-3083.201।.04327.x, indexed in Pubmed: 22077933.

18. Blecken SR, Villavicencio JL, Kao TC. Comparison of elastic versus nonelastic compression in bilateral venous ulcers: a randomized trial. J Vasc Surg. 2005; 42(6): II50-II55, doi: 10.1016/j.jvs.2005.08.015, indexed in Pubmed: 16376207.

19. Blair SD, Wright DD, Backhouse CM, et al. Sustained compression and healing of chronic venous ulcers. BMJ. 1988; 297(6657): | |59-II6I, doi: 10.1136/bmj.297.6657.1159, indexed in Pubmed: 3 | 44330 .

20. Schmid-Schönbein GW, Takase S, Bergan JJ. New advances in the understanding of the pathophysiology of chronic ve- 
nous insufficiency. Angiology. 200I; 52 Suppl I: S27-S34, doi: 10.1177/000331970105200IS04, indexed in Pubmed: II510594.

2I. Bergan JJ, Schmid-Schönbein GW, Smith PD, et al. Chronic venous disease. N Engl J Med. 2006; 355(5): 488-498, doi: 10.1056/NEJMra055289, indexed in Pubmed: 16885552.

22. Tisato V, Zauli G, Voltan R, et al. Endothelial cells obtained from patients affected by chronic venous disease exhibit a pro-inflammatory phenotype. PLoS One. 2012; 7(6): e39543, doi: 10.1371/journal.pone.0039543, indexed in Pubmed: 22737245.

23. Takase $S$, Pascarella $L$, Lerond $L$, et al. Venous hypertension, inflammation and valve remodeling. Eur J Vasc Endovasc Surg. 2004; 28(5): 484-493, doi: 10.1016/j.ejvs.2004.05.012, indexed in Pubmed: 15465369.

24. Castro-Ferreira R, Cardoso R, Leite-Moreira A, et al. The Role of Endothelial Dysfunction and Inflammation in Chronic Venous Disease. Ann Vasc Surg. 2018; 46: 380-393, doi: 10.1016/j. avsg.2017.06.131, indexed in Pubmed: 28688874.

25. Jacob MP, Cazaubon M, Scemama A, et al. Plasma matrix metalloproteinase- 9 as a marker of blood stasis in varicose veins. Circulation. 2002; 106(5): 535-538, doi: 10.1161/01. cir.0000027521.83518.4c, indexed in Pubmed: 12147532.

26. Mannello F, Raffetto JD. Matrix metalloproteinase activity and glycosaminoglycans in chronic venous disease: the linkage among cell biology, pathology and translational research. Am J Transl Res. 201 I; 3(2): 149-158.

27. Mannello F, Ligi D, Canale M, et al. Omics profiles in chronic venous ulcer wound fluid: innovative applications for translational medicine. Expert Rev Mol Diagn. 2014; 14(6): 737-762, doi: 10.1586/14737/59.2014.9273 I2, indexed in Pubmed: 249।8I I9.

28. Kucukguven A, Khalil RA. Matrix metalloproteinases as potential targets in the venous dilation associated with varicose veins. Curr Drug Targets. 2013; 14(3): 287-324, indexed in Pubmed: 23316963.

29. Chen Y, Peng W, Raffetto JD, et al. Matrix Metalloproteinases in Remodeling of Lower Extremity Veins and Chronic Venous Disease. Prog Mol Biol Transl Sci. 2017; 147: 267-299, doi: 10.1016/bs.pmbts.2017.02.003, indexed in Pubmed: 28413031.
30. Serra R, Grande R, Butrico L, et al. Effects of a new nutraceutical substance on clinical and molecular parameters in patients with chronic venous ulceration. Int Wound J. 2016; 13(I): 88-96, doi: I0. I I I/iwj. I 2240, indexed in Pubmed: 246I 2734.

31. Beidler SK, Douillet CD, Berndt DF, et al. Multiplexed analysis of matrix metalloproteinases in leg ulcer tissue of patients with chronic venous insufficiency before and after compression therapy. Wound Repair Regen. 2008; 16(5): 642-648, doi: 10.1 I I I/j.1524-475X.2008.004I5.x, indexed in Pubmed: 19128259.

32. Raffetto JD, Khalil RA. Mechanisms of varicose vein formation: valve dysfunction and wall dilation. Phlebology. 2008; 23(2): 85-98, doi: 10.1258/phleb.2007.007027, indexed in Pubmed: 18453484 .

33. Beidler SK, Douillet CD, Berndt DF, et al. Inflammatory cytokine levels in chronic venous insufficiency ulcer tissue before and after compression therapy. J Vasc Surg. 2009; 49(4): 1013-1020, doi: 10.1016/j.jvs.2008.11.049, indexed in Pubmed: 1934 I889.

34. Murphy MA, Joyce WP, Condron C, et al. A reduction in serum cytokine levels parallels healing of venous ulcers in patients undergoing compression therapy. Eur J Vasc Endovasc Surg. 2002; 23(4): 349-352, doi: 10.1053/ejvs.2002.1597, indexed in Pubmed: I1991698.

35. Gohel MS, Windhaber RAJ, Tarlton JF, et al. The relationship between cytokine concentrations and wound healing in chronic venous ulceration. J Vasc Surg. 2008; 48(5): 1272-1277, doi: 10.1016/j.jvs.2008.06.042, indexed in Pubmed: 18771884 .

36. Beidler SK, Douillet CD, Berndt DF, et al. Multiplexed analysis of matrix metalloproteinases in leg ulcer tissue of patients with chronic venous insufficiency before and after compression therapy. Wound Repair Regen. 2008; 16(5): 642-648, doi: 10.1 III/j.1524-475X.2008.00415.x, indexed in Pubmed: 19128259.

37. Caimi G, Ferrara F, Montana M, et al. Behaviour of the plasma concentration of gelatinases and their tissue inhibitors in subjects with venous leg ulcers. Clin Hemorheol Microcirc. 2015; 60(3): 309-316, doi: 10.3233/CH-141863, indexed in Pubmed: 25I5949I. 